University of Nebraska - Lincoln

DigitalCommons@University of Nebraska - Lincoln

Timothy J. Gay Publications

Research Papers in Physics and Astronomy

May 1999

\title{
Optically Pumped Electron Spin Filter
}

\author{
Herman Batelaan \\ University of Nebraska - Lincoln, hbatelaan@unl.edu
}

A. S. Green

University of Nebraska - Lincoln

B. A. Hitt

University of Nebraska - Lincoln

Timothy J. Gay

University of Nebraska - Lincoln, tgay1@unl.edu

Follow this and additional works at: https://digitalcommons.unl.edu/physicsgay

Part of the Physics Commons

Batelaan, Herman; Green, A. S.; Hitt, B. A.; and Gay, Timothy J. , "Optically Pumped Electron Spin Filter" (1999). Timothy J. Gay Publications. 32.

https://digitalcommons.unl.edu/physicsgay/32

This Article is brought to you for free and open access by the Research Papers in Physics and Astronomy at DigitalCommons@University of Nebraska - Lincoln. It has been accepted for inclusion in Timothy J. Gay Publications by an authorized administrator of DigitalCommons@University of Nebraska - Lincoln. 


\title{
Optically Pumped Electron Spin Filter
}

\author{
H. Batelaan, A. S. Green, B. A. Hitt,* and T. J. Gay \\ Behlen Laboratory of Physics, University of Nebraska, Lincoln, Nebraska 68588-0111
}

(Received 26 October 1998)

\begin{abstract}
This paper reports the first experimental demonstration of an optically pumped electron spin filter. Unpolarized electrons produced in a cold-cathode discharge drift through a mixture of spin-polarized $\mathrm{Rb}$ and a nitrogen or helium buffer gas. Through spin-exchange collisions with the $\mathrm{Rb}$, the drifting electrons become polarized along the optical pumping axis. We study the role of the buffer gas in both the optical pumping and the spin transfer to the free electrons. This spin filter produces electron beams with currents and polarizations comparable to first-generation GaAs polarized electron sources. [S0031-9007(99)09031-6]

PACS numbers: $34.80 .-\mathrm{i}$
\end{abstract}

This Letter reports the experimental demonstration of a new kind of polarized electron source: an optically pumped electron spin filter. Polarized electrons are an indispensable probe of spin-dependent phenomena in many areas of physics [1-3], but they are difficult to produce. State-of-the-art sources of polarized electrons use either photoemission from negative-electron-affinity GaAs (or variants of its basic structure) $[3,4]$ or chemiionization of optically pumped metastable $\mathrm{He}^{*}[5,6]$. Both of these methods can yield average currents on the order of $100 \mu \mathrm{A}$ with polarizations in excess of $70 \%$. Unfortunately, such sources are technically and operationally complex.

In our laboratory, we have been trying to develop "turnkey" sources of polarized electrons, with fewer operating constraints and greater simplicity than the sources just mentioned. Until recently, we had viewed the best candidate for a turnkey source to be one of the type developed by McCusker et al. at Rice University in the early 1970s [7], based on associative ionization of $\mathrm{He}^{*}$. It is different from the $\mathrm{He}^{*}$ source referred to above, in that the electrons are extracted directly from a He discharge instead of being produced by chemi-ionization of the flowing discharge afterglow. While the former design is significantly simpler from the standpoint of mechanical and vacuum engineering, the "flowing afterglow" source produces much higher currents and electron polarizations.

We have attempted to improve the direct extraction source by using newly developed LMA [8] and DBR diode lasers [9] and an improved optical pumping scheme [10] to polarize the $\mathrm{He}^{*}$. These efforts have been unsuccessful [11]. The problem with the direct extraction source is that it relies on having a discharge with a relatively high ratio of metastable atoms to groundstate atoms, a condition we were not able to achieve satisfactorily. The flowing afterglow source solves this problem, but at the expense of a significant increase in source complexity.

If, on the other hand, the primary electron-polarizing collision mechanism involved ground-state atoms instead of excited ones, the problem would be eliminated. This is the idea behind the device we discuss in this Letter: an optically pumped $\mathrm{Rb}$ electron spin filter. Free electrons diffuse under the action of an electric field through $\mathrm{Rb}$ vapor that has been spin polarized by optical pumping. Through spin-exchange collisions with the $\mathrm{Rb}$, the free electrons become polarized and are extracted to form a beam.

The use of spin-exchange collisions to polarize ensembles of electrons is not a new idea. Farago et al. [12] and Krisciokaitis-Krisst et al. [13] developed pulsed sources of polarized electrons (with average currents $<10^{-10} \mathrm{~A}$ ) by directing beams of polarized $\mathrm{Rb}$ and $\mathrm{H}$, respectively, through electron traps. A spin filter is a more efficient electron polarizer than the trap-beam configuration because angular momentum can be transferred to the system much more rapidly by optical pumping. Moreover, the electron densities in a discharge are much higher than in a trap, leading to correspondingly higher currents. Previously, the optically pumped spin-filter concept has been successfully used to polarize beams of cold neutrons in collisions with oriented ${ }^{3} \mathrm{He}$ nuclei [14]. We also mention in this context the experiments of Drouhin et al. [15] and Schönhense and Siegmann [16] in which low-current $(\lessgtr \mathrm{pA})$ beams of electrons have been polarized by passage through magnetized thin solid films.

For effective spin-exchange polarization, $\sigma n_{\mathrm{Rb}} l$ should be of order unity, where $\sigma$ is the spin-exchange cross section for $e^{-}-\mathrm{Rb}$ scattering. As a first estimate, one can calculate the necessary $\mathrm{Rb}$ density, $n_{\mathrm{Rb}}$, for a given interaction path length $l$. If we consider electrons at $5 \mathrm{eV}$, then $\sigma \approx 10^{-15} \mathrm{~cm}^{2}$ [12]. Taking $l=10 \mathrm{~cm}$, this corresponds to $n_{\mathrm{Rb}} \approx 10^{14} \mathrm{~cm}^{-3}$. Pure $\mathrm{Rb}$ vapor with this density is optically thick and cannot be pumped because of radiation trapping and subsequent depolarization [17]. Zeeman shifting of the Rb magnetic sublevels significantly reduces absorption of depolarizing radiation, but magnetic fields of several $\mathrm{kG}$ are required for $n_{\mathrm{Rb}} \sim$ $10^{14} \mathrm{~cm}^{-3}$. A second alternative involves the use of $\mathrm{N}_{2}$ to quench the excited $\mathrm{Rb}$ atoms during the optical pumping 
cycle to reduce emission of depolarizing radiation. This method has been used successfully in alkali spin-exchange polarization of noble gases with $\mathrm{N}_{2}$ pressures of hundreds of Torr [18-21]. The use of buffer gas at such pressures poses another serious experimental challenge insofar as it is necessary to establish a well-defined beam of electrons and to bring it into an experimental chamber. Estimates of the $\mathrm{N}_{2}-\mathrm{Rb}$ quenching cross section, however, imply that significantly lower pressures of $\mathrm{N}_{2}$ might adequately quench the $\mathrm{Rb}$ and lead to reasonable average $\mathrm{Rb}$ polarizations, $P_{\mathrm{Rb}}[20,22]$.

We studied the effect on $P_{\mathrm{Rb}}$ of $\mathrm{N}_{2}$ at pressures below 200 Torr. Some results are shown in Fig. 1. The measurements were made in a stainless steel pipe of length $L=15 \mathrm{~cm}$ with glass windows at both ends to allow the entrance and exit of the optical pumping laser beam as well as a probe beam. The former was produced by a standing-wave dye laser operating at $795 \mathrm{~nm}$, the $D 1$ transition for Rb. The data of Fig. 1 were taken with a laser pump power of $200 \mathrm{~mW}$. Typically less than $50 \%$ of this light was transmitted through the absorption cell. The wavelength selection of the dye laser was made solely with a birefringent crystal in order to maintain a linewidth wider than the broadened $\mathrm{Rb}$ absorption spectrum. An attenuated $3 \mathrm{~mW}$ diode laser probe beam, detuned by a frequency $\delta$ from the $\mathrm{Rb} D 2$ wavelength $\lambda$ of $780 \mathrm{~nm}$, was used to measure $n_{\mathrm{Rb}}$ and $P_{\mathrm{Rb}}$ [18]. The pipe contained a glass ampoule of $\mathrm{Rb}$ with a break-seal stem; the entire apparatus was heated to vary $n_{\mathrm{Rb}}$. Nitrogen of variable pressure was introduced through a needle valve. A longitudinal magnetic field $B$ of up to $600 \mathrm{G}$ could be applied to the center of the pumping region.

At large detuning and strong $B$ fields, the optical rotation of linearly polarized light is dominated by the diamagnetic Faraday effect. Measuring the optical rotation $\Delta \phi$ associated with this effect yields the density

$$
n_{\mathrm{Rb}}=\frac{8 \pi(\Delta \phi) \delta^{2}}{L \Gamma \lambda^{2} \mu_{B} B}
$$

where $\Gamma$ is the $D 2$ natural linewidth, and $\mu_{B}$ is the Bohr magneton. At smaller detunings and lower $B$ fields, the optical rotation is dominated by the paramagnetic Faraday effect, and yields

$$
P_{\mathrm{Rb}}=\frac{56 \pi(\Delta \phi) \delta}{3 n_{\mathrm{Rb}} L \Gamma \lambda^{2}} .
$$

From Fig. 1, it is apparent that when $n_{\mathrm{Rb}} \sim$ $10^{14} \mathrm{~cm}^{-3}, \mathrm{~N}_{2}$ pressures in excess of 50 Torr are necessary for significant polarization, whereas $\mathrm{Rb}$ densities only a factor of 5 lower allow $\sim 70 \%$ polarization with 15 Torr of buffer gas. At $n_{\mathrm{Rb}}=3 \times 10^{12} \mathrm{~cm}^{-3}$, the sample can be fully polarized at $\mathrm{N}_{2}$ pressures below 10 Torr, whereas the same density with no buffer gas would require a magnetic field of several $\mathrm{kG}$ for complete polarization [17]. With a low field and no buffer gas, $P_{\mathrm{Rb}}$ at this density could not be pumped above $10 \%$.
We have made a numerical simulation of the optical pumping process in the test cell, following the basic ideas of Tupa and Anderson [17] and Wagshul and Chupp $[19,23]$. The calculation takes into account the pump laser characteristics, cell geometry, effects of diffusion, buffer gas quenching, and depolarizing collisions. We find qualitative agreement (see Fig. 1) with our experimental results if the buffer gas inhibits diffusion to the walls (where the $\mathrm{Rb}$ is assumed to be completely depolarized) and quenches the $\mathrm{Rb}$. The quenching rate, $R$, of the excited $\mathrm{Rb}$ atoms is taken to be $R=3 \Gamma /\left(3+P_{\mathrm{N}_{2}}\right)$, where $P_{\mathrm{N}_{2}}$ is the nitrogen pressure in Torr [23]. The diffusion coefficient for $\mathrm{Rb}$ in $\mathrm{N}_{2}$ is set at $0.28 \mathrm{~cm}^{2} / \mathrm{s}$ [20]. If either effect is "turned off" in the calculation, the agreement with experiment becomes significantly worse.

These preliminary experimental results and numerical simulations, indicating that significant electron polarization might be achieved with modest $\mathrm{N}_{2}$ and $\mathrm{Rb}$ densities, encouraged us to build a first apparatus, shown in Fig. 2. It consists of a 2.75 " Conflat ${ }^{\circledR}$ nipple with a glass window at one end, attached to a noble gas optical electron polarimeter [24] at the other. As an electron source, we used two coaxial electrodes to maintain a cold-cathode discharge. The outer anode is generally held at ground, with the cathode at several hundred volts. The intense, lightemitting part of the discharge is ring shaped and is well localized between the electrodes. The laser pump beam enters through the window, passes through the electrodes

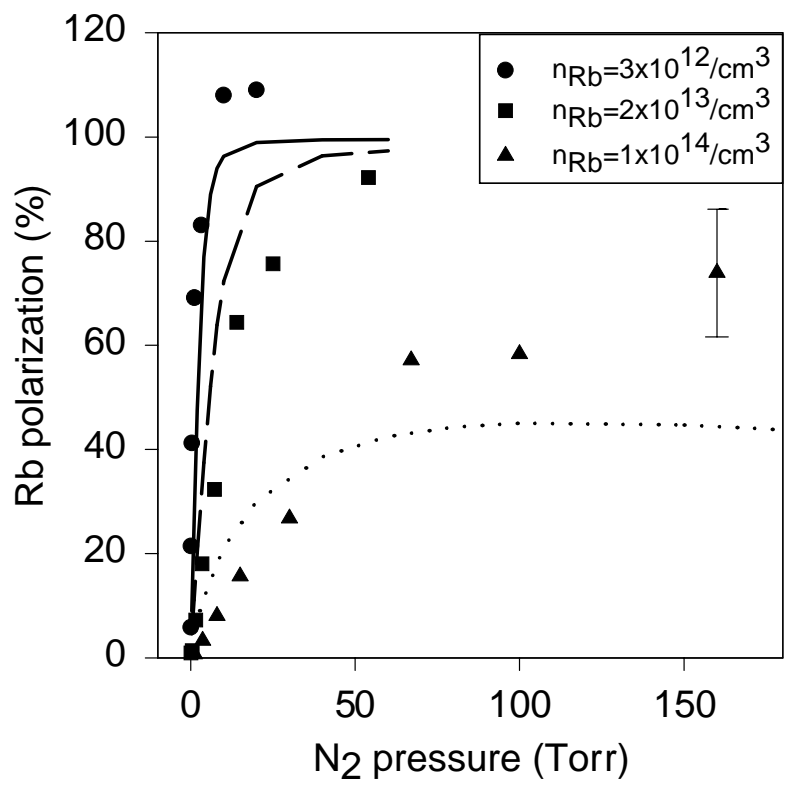

FIG. 1. Rb polarization $\left(P_{\mathrm{Rb}}\right)$ vs nitrogen buffer gas pressure at various $\mathrm{Rb}$ vapor densities. A typical percentage statistical uncertainty is indicated for the high pressure point. Systematic uncertainties in the polarization measurement can be gauged from the fact that $P_{\mathrm{Rb}}$ exceeds unity in the low density data. Results of the numerical simulation for these conditions are also shown with the solid line, dashed line, and dotted line corresponding to the circles, squares, and triangles, respectively. 
and discharge ring, and is partially reflected by a copper extraction aperture that is also electrically isolated. A longitudinal electric field of $\sim 20 \mathrm{~V} / \mathrm{cm}$ established between the cathode and the extraction aperture drives the free electrons through the $\mathrm{Rb} / \mathrm{buffer}$ mixture. As with the test cell, $n_{\mathrm{Rb}}$ can be controlled by heating the source chamber. Nitrogen is admitted from a side port. The laser probe beam enters through the window and passes through the extraction aperture and the optical polarimeter before being detected by a photodiode. During use, the source tube is heated to $\sim 100{ }^{\circ} \mathrm{C}$. Under standard operating conditions, a $5 \mathrm{~g} \mathrm{Rb}$ ampoule lasts two or three weeks. A longitudinal field of up to $600 \mathrm{G}$ is applied to the source and the entrance to the polarimeter.

The polarimeter chamber is made of Pyrex and is pumped with a $1000 \mathrm{l} / \mathrm{s}$ diffusion pump which maintains the vacuum below $10^{-4}$ Torr. Electrons extracted from the source are guided to a region where they collisionally excite an effusive neon target. The Stokes parameters of radiation from the $\mathrm{Ne} 2 p^{5} 3 p^{3} D_{3}$ state are measured with an optical polarimeter, which views the collision region through the side of the chamber. The Stokes parameters can then be used to determine the electron polarization [24]. We discovered that the extracted electron currents were larger and more stable when the $\mathrm{N}_{2}$ pressure was kept quite low. Figure 3 shows data for both $\mathrm{Rb}$ and electron polarization taken with a $\mathrm{N}_{2}$ pressure of 0.4 Torr as the laser power is varied. The appreciable electron polarizations measured in these experiments surprised us, given the low value of $n_{\mathrm{Rb}}$. We attribute the fluctuations in both sets of polarization data to longitudinal mode hopping of the 40-Ghz-wide pump laser.

The rather significant polarizations we observe, an almost certain violation of Murphy's law, are understand-

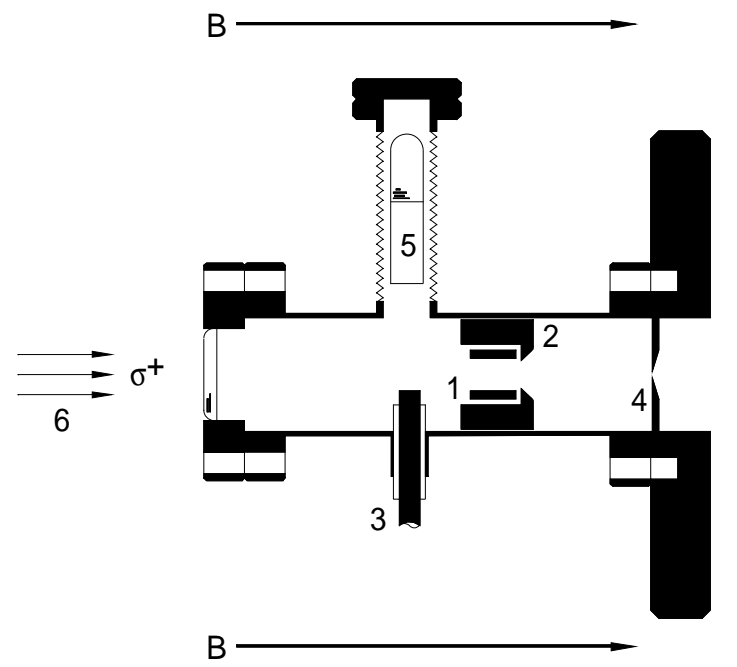

FIG. 2. Diagram of spin filter showing: (1) dc-discharge cold cathode; (2) discharge anode; (3) discharge high-voltage feedthrough; (4) electrically isolated field plate and exit aperture; (5) Rb ampoule; (6) optical pumping radiation. A magnetic field is applied to the entire apparatus. able in terms of the action of the buffer gas. In addition to quenching the excited states of the Rb during optical pumping and inhibiting wall depolarization by acting as a diffusion barrier, the nitrogen buffer gas appears to have other important benefits. First, considering a random walk, the effective path length $l_{\text {eff }}$ of the electrons through the buffer gas increases from $10 \mathrm{~cm}$ (the cell length $L$ ) to $L^{2} / \bar{\lambda}=$ $200 \mathrm{~m}$, where $\bar{\lambda}$ is the mean free path. This means more polarizing collisions can take place. Second, although collisional broadening of the absorption line at about 1 Torr is negligible for optical thicknesses $<1$, the absorption profile at high $\mathrm{Rb}$ densities broadens considerably with a buffer gas [25]; we have measured its FWHM to be much greater than $10 \mathrm{GHz}$ for $P_{\mathrm{N}_{2}}=0.4$ Torr. A wider absorption profile allows more angular momentum to be transferred deeply into the $\mathrm{Rb}$ cell. Third, electron swarm experiments show that a buffer gas will thermalize hot electrons. At $20 \mathrm{~V} / \mathrm{cm}$ and 0.4 Torr of $\mathrm{N}_{2}$ the average electron energy is roughly $1 \mathrm{eV}$ [26]. Since $\sigma$ rises rapidly with decreasing electron energy [12], thermalization of the discharge electrons dramatically improves spin-exchange efficiency. The latter effect also means that the energy spread of the emergent beam is reduced; Ne excitation functions we have measured with the optical polarimeter are consistent with energy spreads of about $1 \mathrm{eV}$ FWHM.

In a final experiment, we tried $\mathrm{He}$ as a discharge/ buffer gas. The results are shown in Fig. 4. We observed that $\mathrm{He}$ discharges were more stable with the electrode geometry and voltages we used. This in combination with

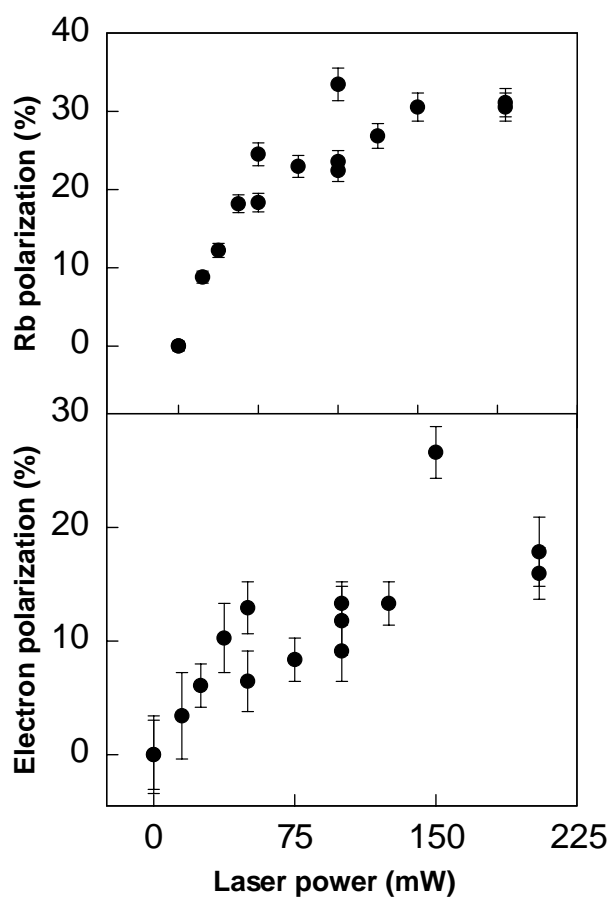

FIG. 3. Rb and electron polarization vs pump laser power with $n_{\mathrm{Rb}}=7 \times 10^{11} \mathrm{~cm}^{-3}$ and a nitrogen buffer pressure of 0.4 Torr. The extracted electron current is $2 \mu \mathrm{A}$. Error bars indicate statistical uncertainty only. 


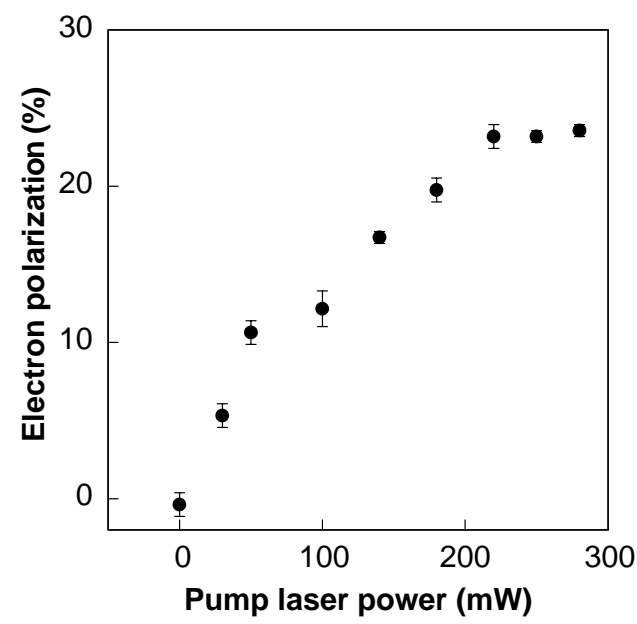

FIG. 4. Electron polarization vs pump laser power with $n_{\mathrm{Rb}}=3 \times 10^{12} \mathrm{~cm}^{-3}$ and a helium buffer pressure of 2 Torr. The extracted electron current is between 4 and $5 \mu \mathrm{A}$.

the fact that these data were taken when the laser was unusually well behaved accounts for the rather "smooth" data set. One interesting aspect of these results is that both $\mathrm{He}$ and $\mathrm{N}_{2}$ yield comparable electron polarizations at pressures of $\sim 1$ Torr. This is not surprising in view of the 3 Torr "cutoff" pressure for the $\mathrm{N}_{2}$ quenching rate, $R$. All other advantages of the buffer gases remain. Note that the electron polarization in the data set of Fig. 3 follows the behavior of $P_{\mathrm{Rb}}$; the former cannot exceed the latter.

In summary, we have demonstrated for the first time an optically pumped electron spin filter. This device holds promise for being a viable turnkey source of polarized electrons. In a crude first version, it has produced electron currents in excess of $2 \mu \mathrm{A}$ with $26 \%$ polarization and an energy width $\leq 1 \mathrm{eV}$. This makes it comparable to typical first-generation GaAs sources [4].

The authors thank B.G. Birdsey, R. J. Knize, M. S. Lubell, and especially P.D. Burrow for useful discussions. This work was supported by the NSF under Grant No. PHY-9732258.

*Present address: J. A. Woolam Co., 645 M Street, Lincoln, NE 68508.

[1] J. Kessler, Polarized Electrons (Springer, Berlin, 1985), 2nd ed.
[2] Polarized Electrons in Surface Physics, edited by R. Feder (World Scientific, Singapore, 1985).

[3] Polarized Gas Targets and Polarized Beams, edited by R. J. Holt and M. A. Miller, AIP Conf. Proc. No. 421 (AIP, New York, 1998).

[4] See, e.g., K. Aulenbacher et al., Nucl. Instrum. Methods Phys. Res., Sect. A 391, 498 (1997), and references therein.

[5] G.H. Rutherford et al., Rev. Sci. Instrum. 61, 1460 (1990).

[6] J. Arianer et al., Nucl. Instrum. Methods Phys. Res., Sect. A 382, 371 (1996).

[7] M. V. McCusker, L. L. Hatfield, and G. K. Walters, Phys. Rev. A 5, 177 (1972).

[8] L. D. Schearer and P. Tin, J. Appl. Phys. 68, 943 (1990).

[9] SDL Model No. SDL-6702-H1.

[10] L. D. Schearer and P. Tin, Phys. Rev. A 42, 4028 (1990).

[11] R. J. Vandiver, Ph.D. thesis, University of Missouri-Rolla, 1990.

[12] P. S. Farago and H.C. Siegmann, Phys. Lett. 20, 279 (1966); D. M. Campbell, H. M. Brash, and P.S. Farago, Proc. R. Soc. Edinb. A 70, 15 (1971).

[13] R. Krisciokaitis-Krisst and W. Y. Tsai, Nucl. Instrum. Methods Phys. Res. 83, 45 (1970); R. Krisciokaitis-Krisst and W. K. Peterson, ibid. 118, 157 (1974).

[14] K. P. Coulter et al., Nucl. Instrum. Methods Phys. Res., Sect. A 288, 463 (1990).

[15] H.-J. Drouhin et al., J. Magn. Magn. Mater. 151, 417 (1995).

[16] G. Schönhense and H. C. Siegmann, Ann. Phys. (Leipzig) 2, 465 (1993).

[17] D. Tupa, L. W. Anderson, D. L. Huber, and J. E. Lawler, Phys. Rev. A 33, 1045 (1986); D. Tupa and L.W. Anderson, ibid. 36, 2142 (1987).

[18] R. J. Knize, Z. Wu, and W. Happer, Adv. At. Mol. Phys. 24, 223 (1988).

[19] T. E. Chupp et al., Phys. Rev. C 36, 2244 (1987).

[20] M.E. Wagshul and T.E. Chupp, Phys. Rev. A 49, 3854 (1994).

[21] W. J. Cummings, O. Häusser, W. Lorenzon, D. R. Swenson, and B. Larson, Phys. Rev. A 51, 4842 (1995).

[22] R. J. Knize (private communication).

[23] M.E. Wagshul and T.E. Chupp, Phys. Rev. A 40, 4447 (1989).

[24] K. W. Trantham, T. J. Gay, and R. J. Vandiver, Rev. Sci. Instrum. 67, 4103 (1996).

[25] P. W. Milonni and J. H. Eberly, Lasers (Wiley, New York, 1988), p. 109.

[26] L. M. Chanin, A. V. Phelps, and M. A. Biondi, Phys. Rev. 128, 219 (1962). 